PROCEEDINGS OF THE

AMERICAN MATHEMATICAL SOCIETY

Volume 131, Number 3, Pages 921-932

S 0002-9939(02)06722-9

Article electronically published on July 17, 2002

\title{
HELLY-TYPE THEOREMS FOR HOMOTHETS OF PLANAR CONVEX CURVES
}

\author{
KONRAD J. SWANEPOEL
}

(Communicated by John R. Stembridge)

\begin{abstract}
Helly's theorem implies that if $\mathcal{S}$ is a finite collection of (positive) homothets of a planar convex body $B$, any three having non-empty intersection, then $\mathcal{S}$ has non-empty intersection. We show that for collections $\mathcal{S}$ of homothets (including translates) of the boundary $\partial B$, if any four curves in $\mathcal{S}$ have non-empty intersection, then $\mathcal{S}$ has non-empty intersection. We prove the following dual version: If any four points of a finite set $S$ in the plane can be covered by a translate [homothet] of $\partial B$, then $S$ can be covered by a translate [homothet] of $\partial B$. These results are best possible in general.
\end{abstract}

\section{Definitions And notation}

We denote the real $d$-dimensional vector space by $\mathbb{R}^{d}$, and call $\mathbb{R}^{2}$ the plane. We denote the convex hull, boundary and interior of a set $S \subseteq \mathbb{R}^{d}$ by conv $S, \partial S$, int $S$, respectively. A set of points $S$ is in convex position if $S \subseteq \partial$ conv $S$. A closed, bounded and convex set $B \subseteq \mathbb{R}^{d}$ with non-empty interior is a convex body. A convex curve $C$ is the boundary $\partial B$ of some convex body $B$ in the plane. We denote the boundary of a triangle by $\Delta$. We only consider segments with distinct endpoints $\boldsymbol{x} \neq \boldsymbol{y}$, denoted by $[\boldsymbol{x y}]$. A wedge is the union of two non-parallel segments with a common endpoint, i.e., $[\boldsymbol{a} \boldsymbol{b}] \cup[\boldsymbol{b} \boldsymbol{c}]$ for some non-collinear $\boldsymbol{a}, \boldsymbol{b}, \boldsymbol{c}$. A convex curve $C$ is strictly convex if it contains no segment $[\boldsymbol{x y}]$. The line through $\boldsymbol{x}$ and $\boldsymbol{y}$ is denoted by $\overleftrightarrow{\boldsymbol{x y}}$. An affine diameter of a convex curve $C$ in the direction $\boldsymbol{v}$ is a segment $[\boldsymbol{a} \boldsymbol{b}]$ parallel to $\boldsymbol{v}$ with $\boldsymbol{a}, \boldsymbol{b} \in C$ such that no other segment parallel to $\boldsymbol{v}$ with endpoints on $C$ is longer than $[\boldsymbol{a b}]$.

A translate of a set $S$ is a set of the form $\boldsymbol{v}+S$ for some $\boldsymbol{v} \in \mathbb{R}^{2}$. A (positive) homothet of $S$ with homothety factor $\lambda>0$ is a set of the form $\boldsymbol{v}+\lambda S$ for some $\boldsymbol{v} \in \mathbb{R}^{2}$. (Thus we do not allow negative homothets, but allow translates.) For $S \subseteq \mathbb{R}^{d}$, let $\mathcal{H}_{S}$ denote the collection of homothets of $S, \mathcal{T}_{S}$ the collection of translates of $S$, and $\mathcal{H}_{S}^{(\varepsilon)}$ the collection of homothets of $S$ with homothety factor in the interval $[1,1+\varepsilon]$.

The size of a finite set $A$ is denoted by \#A. The following definitions are modifications of the congruence index introduced by Blumenthal [2, §37]. A collection $\mathcal{S}$ has Helly index $(n, k)$ if any finite sub-collection $\mathcal{T} \subseteq \mathcal{S}$ of size $\# \mathcal{T}>n+k$ has

Received by the editors October 12, 2000 and, in revised form, October 23, 2001.

2000 Mathematics Subject Classification. Primary 52A23; Secondary 52A10.

Key words and phrases. Helly-type theorem, convex curves, congruence index, congruence indices. 
non-empty intersection, provided any $n$ sets in $\mathcal{T}$ have non-empty intersection. If $\mathcal{S}$ has Helly index $(n, 0)$ we say that it has Helly order $n$.

A collection $\mathcal{S}$ has Menger index $(n, k)$ if any finite set $S$ of size $\# S>n+k$ is contained in some set of $\mathcal{S}$ whenever each subset of $S$ of $n$ elements is contained in some set of $\mathcal{S}$. If $\mathcal{S}$ has Menger index $(n, 0)$ we say that it has Menger order $n$.

For the sake of simplicity we only consider finite sub-collections $\mathcal{T}$ and finite sets $S$ in these definitions. Although the word "finite" may be removed from the definition of Helly index in Theorem A below by a compactness argument, it is not possible to remove "finite" from the definition of Menger index in Theorem B.

\section{INTRODUCTION}

2.1. Motivation. A Helly-type theorem is an analogue of

Helly's theorem ([12]). The collection of convex sets in $\mathbb{R}^{d}$ has Helly order $d+1$.

See the surveys [4] and 6] for collections of Helly-type theorems. Most of them deal with collections of convex sets. Notable exceptions are the theorem of Amenta [1] (also known as the theorem of Morris [6]) on disjoint unions of convex sets, the theorem of Motzkin [16, 5] on collections of algebraic varieties, and the theorem of Maehara [14, 7] on spheres. In [17] the author proved a Helly-type result for collections of boundaries of axis-aligned boxes and in [18] for boundaries of convex polygons in the plane. Note that finding the smallest Helly order of $\boldsymbol{\mathcal { T }}_{B}$ for a given convex body $B$ is known as the Szökefalvi-Nagy problem, and is known only for a few classes of convex bodies (see [3, Chapter 4]).

A Helly-type theorem can also concern coverings instead of intersections, an example of which is the following consequence of Helly's theorem, noted by Vincensini and Klee [6].

Vincensini-Klee theorem. Let $B$ be a convex body in $\mathbb{R}^{d}$. Then $\mathcal{T}_{B}$ has Menger order $d+1$.

To deduce this theorem from Helly's theorem, we only need the following simple

Lemma 1. For any $S \subseteq \mathbb{R}^{d}, \mathcal{T}_{S}$ has Helly index $(n, k)$ iff $\mathcal{T}_{S}$ has Menger index $(n, k)$.

Proof. The lemma follows from the following observation: Given $\boldsymbol{x}_{1}, \ldots, \boldsymbol{x}_{m} \in \mathbb{R}^{d}$, we have that

$$
\boldsymbol{x} \in \bigcap_{i=1}^{m}\left(\boldsymbol{x}_{i}+S\right) \Longleftrightarrow\left\{\boldsymbol{x}_{1}, \ldots, \boldsymbol{x}_{m}\right\} \subseteq \boldsymbol{x}-S .
$$

Thus $\left\{\boldsymbol{x}_{i}+S: i=1, \ldots, m\right\}$ has non-empty intersection iff $\left\{\boldsymbol{x}_{1}, \ldots, \boldsymbol{x}_{m}\right\}$ can be covered by a translate of $-S$. It follows that $\boldsymbol{\mathcal { T }}_{S}$ has Menger index $(n, k)$ iff $\boldsymbol{\mathcal { T }}_{-S}$ (or equivalent, $\mathcal{T}_{S}$ ) has Helly index $(n, k)$.

In this paper we study Helly and Menger indices for translates and homothets of convex curves in the plane. We now give an overview of previously known results, and then discuss our results and open problems.

2.2. Previous results. The following result, especially in the case of circles, must be a very old observation. See [2, §61] for the case of circles and [13, 8, 9] for the case of strictly convex curves. 


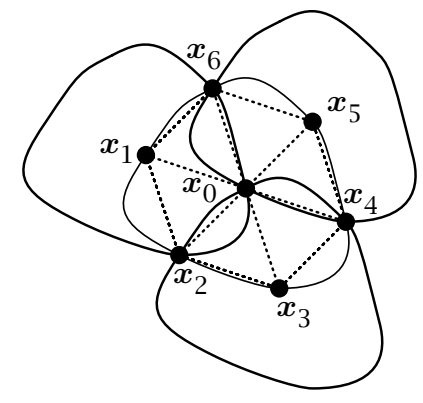

Figure 1. Convex curve with inscribed hexagon

Proposition. For any convex curve $C, \mathcal{T}_{C}$ does not have Helly order 3 . Hence $\mathcal{H}_{C}$ does not have Helly order 3 and, by Lemma囵, $\mathcal{T}_{C}$ does not have Menger order 3 .

Proof. Find an affine regular hexagon inscribed in $C$, with vertices $\boldsymbol{x}_{1}, \ldots, \boldsymbol{x}_{6}$, in this order, and centre $\boldsymbol{x}_{0}$. (There always exists such a hexagon [11.) See Figure 1 Then the required four translates are $C, C+\boldsymbol{x}_{1}-\boldsymbol{x}_{0}, C+\boldsymbol{x}_{3}-\boldsymbol{x}_{0}, C+\boldsymbol{x}_{5}-\boldsymbol{x}_{0}$.

The following result is proved in [2, §61] for circles and [13] for strictly convex smooth curves (although their proof does not use smoothness), and [8, 9] in general, where stronger results are also shown. The proof given below highlights the combinatorial character of the result.

Proposition. For any strictly convex curve $C, \mathcal{H}_{C}$ has Helly index $(3,1)$.

Proof. The statement is a purely combinatorial consequence of the well-known fact that two distinct homothets of a convex curve intersect in at most two points (see also Lemma 2 below). The result follows from the the following easily proved lemma.

Lemma. Let $\mathcal{S}$ be a collection of sets such that $\#\left(S_{1} \cap S_{2}\right) \leq 2$ for all distinct $S_{1}, S_{2} \in \mathcal{S}$, and $\#\left(S_{1} \cap S_{2} \cap S_{3}\right) \geq 1$ for all distinct $S_{1}, S_{2}, S_{3} \in \mathcal{S}$. Then $\bigcap \mathcal{S} \neq \emptyset$, except if $\# \mathcal{S}=4$ and there exists a set of four elements $X=\left\{x_{1}, x_{2}, x_{3}, x_{4}\right\}$ such that $\{S \cap X: S \in \mathcal{S}\}=\left\{X \backslash\left\{x_{i}\right\}: i=1,2,3,4\right\}$.

Getmanenko [8, 9] also has similar results for unbounded convex curves, and refinements of the above result.

Proposition (Getmanenko [8, 9]). For any convex curve $C, \mathcal{H}_{C}$ has Helly order 6. Hence $\mathcal{T}_{C}$ has Helly and Menger orders 6.

\subsection{New results.}

Theorem A. For any convex curve $C$ in the plane, $\mathcal{H}_{C}$ has Helly order 4.

There exist convex curves $C$ for which even $\mathcal{T}_{C}$ does not have Helly index $(3, k)$ for any $k$. Call a convex curve $C$ flat if it contains two parallel segments on different supporting lines. Also, call $C$ thin if $C$ contains a segment $[\boldsymbol{a b}]$ on some line $\ell$, and such that the line equidistant from $\ell$ and $\ell^{\prime}$ intersects $C$ in two points $\boldsymbol{c}$ and $\boldsymbol{d}$ such that $\boldsymbol{c d}<\boldsymbol{a b}$, where $\ell^{\prime} \neq \ell$ is a supporting line of $C$ parallel to $\ell$. See Figure 5 

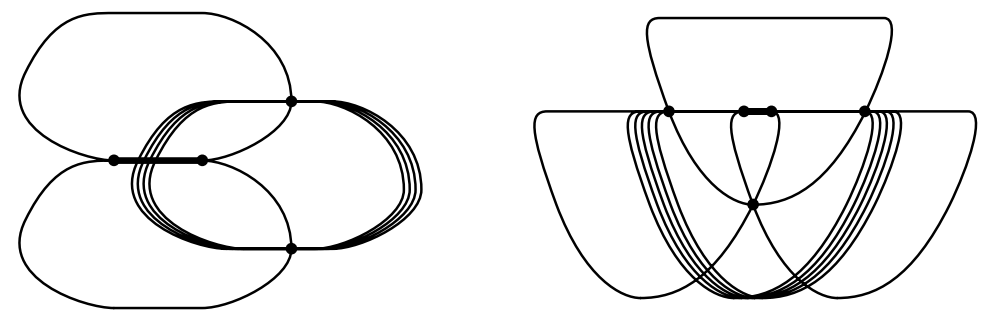

FiguRe 2. For any flat or thin curve there are infinitely many translates with non-empty intersection although any three intersect

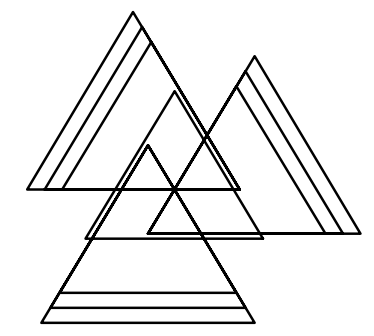

Figure 3. For any triangular curve there are infinitely many homothets with non-empty intersection although any three intersect

Proposition 1. Let $C$ be a convex curve in the plane.

1. If $C$ is flat or thin but is not a triangle, then $\mathcal{T}_{C}$ does not have Helly index $(3, k)$ for any $k \in \mathbb{N}$.

2. The collection $\mathcal{T}_{\Delta}$ has Helly index $(3,1)$, but $\mathcal{H}_{\Delta}^{(\varepsilon)}$ does not have Helly index $(3, k)$ for any $k \in \mathbb{N}$ and $\varepsilon>0$.

See Figures 2 and 3 for examples.

Let $\mathcal{E}$ be the class of convex curves $C$ that are neither flat nor thin.

Corollary 1. If $\boldsymbol{\mathcal { T }}_{C}$ has Helly index $(3,1)$, then either $C$ is a triangle or $C \in \mathcal{E}$.

The following conjecture would settle the question of the best Helly index for homothets or translates of any convex curve.

Conjecture 1. If $C \in \mathcal{E}$, then $\mathcal{H}_{C}$ has Helly index $(3,1)$.

In proving Theorem it is convenient to first prove a covering version, i.e., a theorem about the Menger order of homothets of a convex curve.

Theorem B. For any convex curve $C$ in the plane, $\mathcal{H}_{C}$ has Menger order 4.

This theorem is best possible in the following strong sense:

Proposition 2. For any convex curve $C$ except a triangle and any $\varepsilon>0, \mathcal{H}_{C}^{(\varepsilon)}$ does not have Menger index $(3, k)$ for any $k \in \mathbb{N}$.

Also, $\mathcal{H}_{\Delta}^{(1 / 2)}$ does not have Menger index $(3, k)$ for any $k \in \mathbb{N}$.

We remark that $\mathcal{H}_{\Delta}^{(\varepsilon)}$ has Menger index $(3,1)$ for any $\varepsilon<1 / 2$. We omit the simple proof. 
Applying Lemma 1 to Theorem B and Proposition 1 we obtain the following:

Proposition 3. Let $C$ be a convex curve in the plane.

1. The collection $\mathcal{T}_{C}$ has Menger order 4 .

2. If $C$ is flat or thin but is not a triangle, then $\mathcal{T}_{C}$ does not have Menger index $(3, k)$ for any $k \in \mathbb{N}$.

3. $\mathcal{T}_{\Delta}$ has Menger index $(3,1)$.

From Conjecture 1 the following would follow, completing the picture for translates.

Conjecture 2. If $C \in \mathcal{E}$, then $\mathcal{T}_{C}$ has Menger index $(3,1)$.

It remains to prove Propositions 1 and 2 (Section 4) and Theorems $\mathrm{A}$ and B (Section 5). Note that the proofs of Theorems $\mathrm{A}$ and $\mathrm{B}$, although elementary, are quite intricate when compared to e.g. the proof of Helly's Theorem in $\mathbb{R}^{2}$. Before the proofs we do some geometric preparation in Section 3

We conclude the Introduction with the following remarks. First, in dimensions higher than two it seems that only for very restricted classes of convex bodies $B$ (such as polytopes or certain semi-algebraic sets) would $\mathcal{H}_{\partial B}$ or $\mathcal{T}_{\partial B}$ have finite Helly or Menger orders.

Second, we may also consider the collection $\mathcal{C}_{S}$ of congruent copies of a set $S$. Getmanenko [8] showed that for many compact sets $B$ with non-empty interior in $\mathbb{R}^{d}$, as well as the $k$-skeletons of convex polytopes, with $2 \leq k \leq d, \mathcal{C}_{B}$ does not have a finite Menger order. Blumenthal [2] §61] showed that if $C$ is the boundary of a regular $n$-gon in the plane, then $\mathcal{C}_{C}$ has Menger order $2 n+1$ with Getmanenko [8] improving this to $2 n$ for $n$ sufficiently large. Getmanenko furthermore shows that for the boundary $C$ of any convex $n$-gon, $\mathcal{C}_{C}$ has Menger order $2 n+6$. It would be interesting to characterize those planar convex curves $C$ such that $\mathcal{C}_{C}$ has finite Helly or Menger orders. See [19] for examples of curves $C$ such that $\mathcal{C}_{C}$ does not have Menger index $(3,1)$.

\section{The GeOMEtRy of homothets of CONVEX CuRVES}

Here we discuss basic facts about the covering of sets by homothets of a convex curve and the intersection of homothets of a convex curve.

The following technical lemma is already mentioned in e.g. [10]. A detailed proof may be found in [15, p. 107]. See also [9, Lemma 5].

Lemma 2. Let $T=\left\{\boldsymbol{x}_{1}, \boldsymbol{x}_{2}, \boldsymbol{x}_{3}\right\} \subset \mathbb{R}^{2}$ be any three points. Let $T^{\prime}=\left\{\boldsymbol{x}_{1}^{\prime}, \boldsymbol{x}_{2}^{\prime}, \boldsymbol{x}_{3}^{\prime}\right\}$ be a homothet of $T$. If $T \cup T^{\prime}$ is in convex position, then either $T$ is collinear, or for some $i,\left(T \cup T^{\prime}\right) \backslash\left\{\boldsymbol{x}_{i}, \boldsymbol{x}_{i}^{\prime}\right\}$ is collinear.

Lemma 3. Let $T=\left\{\boldsymbol{x}_{1}, \boldsymbol{x}_{2}, \boldsymbol{x}_{3}\right\}$ be a non-collinear set and $C$ a convex curve in the plane that is not a triangle homothetic to $\partial \operatorname{conv} T$. If $T$ is contained in more than one homothet of $C$, then there exist distinct $i, j$ (depending only on $T$ and $C$ ) such that any homothet $C^{\prime}$ of $C$ containing $T$ also contains $\left[\boldsymbol{x}_{i} \boldsymbol{x}_{j}\right]$.

Proof. By Lemma2, if $T \subseteq C^{\prime} \cap C^{\prime \prime}\left(C^{\prime}\right.$ and $C^{\prime \prime}$ distinct homothets of $\left.C\right)$, then there are distinct $i, j$ such that $\left[\boldsymbol{x}_{i} \boldsymbol{x}_{j}\right] \subseteq C^{\prime} \cap C^{\prime \prime}$. Suppose that for each choice of distinct $i, j$, there is a homothet $C_{i j}$ of $C$ such that $T \subset C_{i j}$, but $\left[\boldsymbol{x}_{i} \boldsymbol{x}_{j}\right] \cap C_{i j}=\left\{\boldsymbol{x}_{i}, \boldsymbol{x}_{j}\right\}$. Then we obtain $\left[\boldsymbol{x}_{1} \boldsymbol{x}_{3}\right] \subseteq C_{12} \cap C_{23},\left[\boldsymbol{x}_{2} \boldsymbol{x}_{3}\right] \subseteq C_{12} \cap C_{13},\left[\boldsymbol{x}_{1} \boldsymbol{x}_{2}\right] \subseteq C_{13} \cap C_{23}$. It follows that $C_{12}$ contains the wedge $\left[\boldsymbol{x}_{1} \boldsymbol{x}_{3}\right] \cup\left[\boldsymbol{x}_{2} \boldsymbol{x}_{3}\right]$. Hence $C$ contains a homothet 

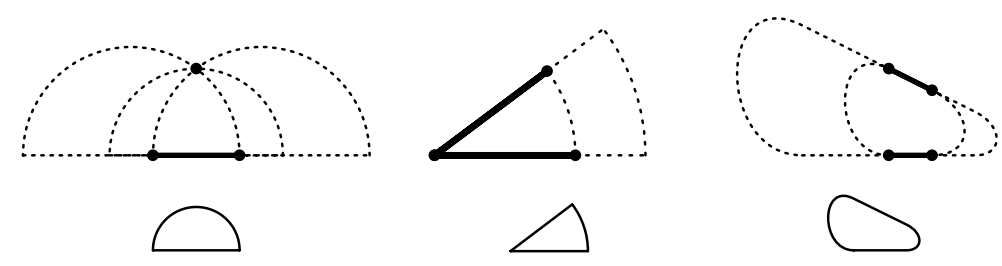

FIgURE 4. The intersection of homothets of a convex closed curve

of this wedge. Similarly, $C$ contains two other such wedges, and it follows that $C$ is homothetic to $\partial \operatorname{conv} T$.

It is readily seen that Lemma 3 does not hold for a triangle homothetic to $\partial \operatorname{conv} T$.

The following lemma describes the intersection of a collection of homothets of a convex curve. Note that although the general case is interesting in its own right, for the purposes of this paper we only need to know how two homothets intersect (see [15, §3.3] for a discussion of the history of this special case).

Lemma 4. Let $C$ be a convex closed curve in the plane. The intersection of at least two distinct homothets of $C$ is the union of two sets, each of which is either a segment, a singleton or empty (Figure 4), unless $C$ is the boundary of a triangle and the number of homothets is more than two, in which case the intersection can also be a set of three points homothetic to the vertex set of $C$.

Proof. We assume that $C$ is not the boundary of a triangle, as this case is simple. Let $\mathcal{S}$ be a collection of at least two homothets of $C$. Note that $\cap \mathcal{S}$ is in convex position, since it is contained in a convex curve. If $\bigcap \mathcal{S}$ is collinear, then it is clearly either empty, a singleton, two points or a segment. Thus without loss of generality let $\boldsymbol{a}, \boldsymbol{b}, \boldsymbol{c}$ be three non-collinear points in $\bigcap \mathcal{S}$. By Lemma 3 we have without loss of generality that $[\boldsymbol{b c}] \subseteq \bigcap \mathcal{S}$. Also assume without loss of generality that $[\boldsymbol{b} \boldsymbol{c}]$ is a maximal segment in $\bigcap \mathcal{S}$, i.e., $\cap \mathcal{S} \cap \overleftrightarrow{\boldsymbol{b c}}=[\boldsymbol{b c}]$.

If there is a point of $\bigcap \mathcal{S}$ on $\overleftrightarrow{\boldsymbol{a c}}$ except $\boldsymbol{a}$ or $\boldsymbol{c}$, then $[\boldsymbol{a c}] \subseteq \bigcap \mathcal{S}$; again assume that $[\boldsymbol{a c}]$ is a maximal segment in $\bigcap \mathcal{S}$, and choose an $\boldsymbol{x}$ between $\boldsymbol{a}$ and $\boldsymbol{c}$, and a $\boldsymbol{y}$ between $\boldsymbol{b}$ and $\boldsymbol{c}$. Then $\boldsymbol{x}, \boldsymbol{y}$, and any point in $\bigcap \mathcal{S} \backslash([\boldsymbol{a c}] \cup[\boldsymbol{b c}])$ would contradict Lemma 3, It follows that $\bigcap \mathcal{S}=[\boldsymbol{a c}] \cup[\boldsymbol{b} \boldsymbol{c}]$.

We may now assume that $\bigcap \mathcal{S} \cap \overleftrightarrow{\boldsymbol{a c}}=\{\boldsymbol{a}, \boldsymbol{c}\}$, and similarly, $\cap \mathcal{S} \cap \overleftrightarrow{\boldsymbol{b c}}=\{\boldsymbol{b}, \boldsymbol{c}\}$. Then for any $\boldsymbol{d} \in \bigcap \mathcal{S} \backslash(\{\boldsymbol{a}\} \cup[\boldsymbol{b} \boldsymbol{c}])$ we have that $\{\boldsymbol{a}, \boldsymbol{b}, \boldsymbol{c}, \boldsymbol{d}\}$ is in convex position, since $\cap \mathcal{S}$ is in convex position. If $[\boldsymbol{c d}] \subset \cap \mathcal{S}$, then Lemma 3 will be contradicted by a point between $\boldsymbol{c}$ and $\boldsymbol{d}$, a point between $\boldsymbol{b}$ and $\boldsymbol{c}$, and $\boldsymbol{a}$. Since $[\boldsymbol{a c}] \nsubseteq \cap \mathcal{S}$, by Lemma 3 it now follows that $[\boldsymbol{a d}] \subset \cap \mathcal{S}$. Assume without loss of generality that $[\boldsymbol{a d}]$ is a maximal segment in $\bigcap \mathcal{S}$. Then a point in $\bigcap \mathcal{S} \backslash([\boldsymbol{a d}] \cup[\boldsymbol{b c}])$, a point between $\boldsymbol{a}$ and $\boldsymbol{d}$, and a point between $\boldsymbol{b}$ and $\boldsymbol{c}$, contradicts Lemma 3. It follows that $\bigcap \mathcal{S}=[\boldsymbol{a d}] \cup[\boldsymbol{b} \boldsymbol{c}]$.

\section{The EXAMPles}

Proof of Proposition 1. We omit the simple proof that $\mathcal{T}_{\Delta}$ has Menger and Helly indices $(3,1)$. Figure 3 shows that $\mathcal{H}_{\Delta}^{(\varepsilon)}$ does not have Helly index $(3, k)$ for any $k \in \mathbb{N}$. 


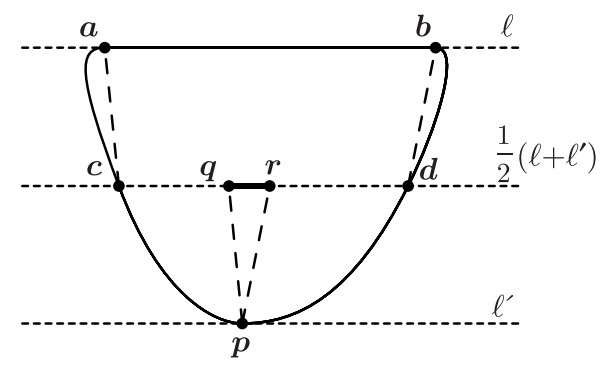

FIGURE 5. A thin convex curve

Now let $C$ be a flat convex curve. We show that $\mathcal{T}_{C}$ does not have Menger index $(3, k)$. We may assume without loss that $[(\boldsymbol{x}-\alpha \boldsymbol{v})(\boldsymbol{x}+\alpha \boldsymbol{v})],[(\boldsymbol{y}-\beta \boldsymbol{v})(\boldsymbol{y}+\beta \boldsymbol{v})] \subseteq C$, where $\boldsymbol{y}-\boldsymbol{x}$ and $\boldsymbol{v}$ are linearly independent, $0<\alpha \leq \beta$, and $\alpha, \beta$ maximal. Then there exist continuous functions $f, g:[0,1] \rightarrow \mathbb{R}$ such that $f, g>0$ and

$$
\begin{aligned}
C= & {[(\boldsymbol{x}-\alpha \boldsymbol{v})(\boldsymbol{x}+\alpha \boldsymbol{v})] \cup[(\boldsymbol{y}-\beta \boldsymbol{v})(\boldsymbol{y}+\beta \boldsymbol{v})] } \\
& \cup\{(1-\lambda) \boldsymbol{x}+\lambda \boldsymbol{y}+f(\lambda) \boldsymbol{v}: 0 \leq \lambda \leq 1\} \\
& \cup\{(1-\lambda) \boldsymbol{x}+\lambda \boldsymbol{y}-g(\lambda) \boldsymbol{v}: 0 \leq \lambda \leq 1\} .
\end{aligned}
$$

Choose $\varepsilon \in(0,1)$ such that $f(\epsilon)=g(1-\varepsilon)$. This is possible since $f(0)-g(1)=$ $\alpha-\beta \leq 0$ and $f(1)-g(0)=\beta-\alpha \geq 0$. Let $S=\{\boldsymbol{x}, \boldsymbol{y}\} \cup[(\boldsymbol{z}-\alpha \boldsymbol{v})(\boldsymbol{z}+\alpha \boldsymbol{v})]$, where $\boldsymbol{z}=(1-\varepsilon) \boldsymbol{x}+\varepsilon \boldsymbol{y}+f(\epsilon) \boldsymbol{v} \in C$. That any three points of $S$ are covered by some translate of $C$ follows from the following easily verified facts:

1. $\{\boldsymbol{x}, \boldsymbol{y}, \boldsymbol{z}+\gamma \boldsymbol{v}\} \subseteq C+\gamma \boldsymbol{v}$ for all $\gamma \in[-\alpha, \alpha]$,

2. $\{\boldsymbol{x}\} \cup[(\boldsymbol{z}-\alpha \boldsymbol{v})(\boldsymbol{z}+\alpha \boldsymbol{v})] \subseteq C+(1-\varepsilon)(\boldsymbol{x}-\boldsymbol{y})+f(\varepsilon) \boldsymbol{v}$,

3. $\{\boldsymbol{y}\} \cup[(\boldsymbol{z}-\alpha \boldsymbol{v})(\boldsymbol{z}+\alpha \boldsymbol{v})] \subseteq C+\varepsilon(\boldsymbol{y}-\boldsymbol{x})+f(\varepsilon) \boldsymbol{v}$.

Finally, note that $S$ is not in convex position, hence is not coverable by any translate of $C$ (Figure 2).

Now let $C$ be a thin convex curve that is not a triangle. Let $C$ contain the segment $[\boldsymbol{a} \boldsymbol{b}]$ on line $\ell$, with no other segment parallel to $\ell$. Let $\ell^{\prime} \neq \ell$ be a line parallel to $\ell$, supporting $C$. Let the line $\frac{1}{2}\left(\ell+\ell^{\prime}\right)$ equidistant from $\ell$ and $\ell^{\prime}$ intersect $C$ in $\boldsymbol{c}$ and $\boldsymbol{d}$. Let $\ell^{\prime}$ intersect $C$ in $\boldsymbol{p}$. Let $\boldsymbol{q}=\boldsymbol{p}+\boldsymbol{a}-\boldsymbol{c}$ and $\boldsymbol{r}=\boldsymbol{p}+\boldsymbol{b}-\boldsymbol{d}$. Then $S=\{\boldsymbol{p}, \boldsymbol{c}, \boldsymbol{d}\} \cup[\boldsymbol{q} \boldsymbol{r}]$ is the desired set. See Figures 5 and 2 .

Proof of Proposition 2. We first describe an $S \subseteq \mathbb{R}^{2}$ of size $>3+k$ showing that $\mathcal{H}_{\Delta}^{(1 / 2)}$ does not have Menger index $(3, k)$ for any $k$. Let the vertices of $\Delta$ be $\boldsymbol{a}_{1}, \boldsymbol{a}_{2}, \boldsymbol{a}_{3}$. Let $\boldsymbol{b}_{i}=\frac{1}{3} \boldsymbol{a}_{i}+\frac{2}{3} \boldsymbol{a}_{i+1}, \boldsymbol{c}_{i}=\frac{2}{3} \boldsymbol{a}_{i}+\frac{1}{3} \boldsymbol{a}_{i+1}$ for all $i$, with indices modulo 3 . Let $\boldsymbol{c}=\frac{1}{3}\left(\boldsymbol{a}_{1}+\boldsymbol{a}_{2}+\boldsymbol{a}_{3}\right)$. Let $S^{\prime}$ be an arbitrary subset of at least $3+k$ elements of $\left[\boldsymbol{b}_{1} \boldsymbol{c}_{1}\right] \cup\left[\boldsymbol{b}_{2} \boldsymbol{c}_{2}\right] \cup\left[\boldsymbol{b}_{3} \boldsymbol{c}_{3}\right]$ such that $S^{\prime}$ contains at least one element of each $\left[\boldsymbol{b}_{i} \boldsymbol{c}_{i}\right]$. Then $S=\{c\} \cup S^{\prime}$ is the required set.

Now let $C$ be a convex curve that is not a triangle. By the proof of Proposition! we already know that if $C$ is the boundary of a parallelogram, then $\mathcal{T}_{C}$ does not have Menger index $(3, k)$ for any $k \in \mathbb{N}$. If $C$ is not a triangle or a parallelogram, then it is easily seen that $C$ contains an arc $\Gamma$ that is not contained in the union of two segments, and such that no chord of $\Gamma$ is an affine diameter of $C$. Let the endpoints of $\Gamma$ be $\boldsymbol{a}$ and $\boldsymbol{b}$. Let $\boldsymbol{p}$ be a point inside $C$ sufficiently near the boundary (depending on $\varepsilon$ ). Let $S=\Gamma \cup\{\boldsymbol{p}\}$. Consider any two points $\boldsymbol{c}, \boldsymbol{d} \in \Gamma$ together with $\boldsymbol{p}$. By enlarging this subset slightly such that the images of $\boldsymbol{c}$ and $\boldsymbol{d}$ stay on 


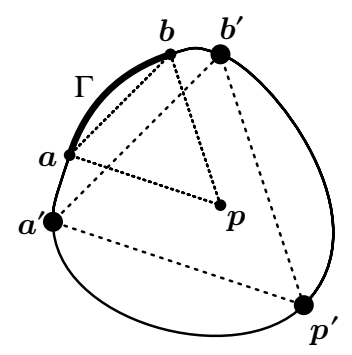

Figure 6. Proof of Proposition 2

$C$, the image of $\boldsymbol{p}$ eventually hits $C$. Since $\boldsymbol{p}$ is sufficiently near the boundary, this happens before the homothety ratio exceeds $1+\varepsilon$. See Figure 6. Thus any three points of $S$ can be covered by a homothet in $\mathcal{H}_{C}^{(\varepsilon)}$.

If $S$ can be covered by some homothet of $C$, then $\Gamma$ would be contained in the intersection of two distinct homothets of $C$, hence contained in the union of two (possibly degenerate) segments, by Lemma 4 a contradiction. Thus $S$ cannot be covered by any homothet of $C$.

We can modify this construction to make $S$ finite by choosing at least $3+k$ points on $\Gamma$ that still cannot be covered by the union of two segments.

\section{Proofs of the theorems}

Proof of Theorem $B$. We omit the simple proof that the Menger order of $\mathcal{H}_{\Delta}$ is 4 , and assume for the remainder of the proof that $C$ is not the boundary of a triangle. Assume that $\# S>4$ and that any four points in $S$ can be covered by a homothet of $C$. Assume that $S$ is non-collinear, as the theorem is otherwise trivial. We now apply the following theorem of Steinitz (see [6]):

Let $\boldsymbol{p} \in \mathbb{R}^{d}$ and $S \subseteq \mathbb{R}^{d}$. If $\boldsymbol{p} \in \operatorname{int}$ conv $S$, then $\boldsymbol{p} \in \operatorname{int}$ conv $S^{\prime}$ for some $S^{\prime} \subseteq S$ with \# $S^{\prime} \leq 2 d-1$, except if $S=\left\{\boldsymbol{p}+\lambda_{i} \boldsymbol{e}_{i}, \boldsymbol{p}-\mu_{i} \boldsymbol{e}_{i}: i=1, \ldots, d\right\}$ for some $\lambda_{i}, \mu_{i}>0$ and some basis $\boldsymbol{e}_{1}, \ldots, \boldsymbol{e}_{d}$ of $\mathbb{R}^{d}$.

Since any four points of $S$ are in convex position, if $S$ is not in convex position, then $S$ consists of 5 points $\{\boldsymbol{a}, \boldsymbol{b}, \boldsymbol{c}, \boldsymbol{d}, \boldsymbol{e}\}$ with $\boldsymbol{e}$ between $\boldsymbol{a}$ and $\boldsymbol{c}$, and between $\boldsymbol{b}$ and $\boldsymbol{d}$. By considering how homothets of $C$ cover any 4 points of $S$, we obtain that $C$ has two supporting lines parallel to $\boldsymbol{a c}$, and similarly, two supporting lines parallel to $\boldsymbol{b} \boldsymbol{d}$. We may therefore circumscribe a parallelogram around $C$, with a side parallel to $\boldsymbol{a c}$ (of length $r$, say), and a side parallel to $\boldsymbol{b} \boldsymbol{d}$ (of length $s$, say). Since $\{\boldsymbol{a}, \boldsymbol{b}, \boldsymbol{c}, \boldsymbol{d}\}$ can be covered by a homothet of $C$, we have $\boldsymbol{a c} / r=\boldsymbol{b} \boldsymbol{d} / s$. Since $\{\boldsymbol{a}, \boldsymbol{c}, \boldsymbol{d}, \boldsymbol{e}\}$ can be covered by a homothet of $C, \boldsymbol{a c} / r \leq \boldsymbol{d e} / s$. Similarly, $\boldsymbol{a c} / r \leq \boldsymbol{b e} / s$. It follows that $\boldsymbol{b} \boldsymbol{d} / s \geq 2 \boldsymbol{a c} / r$, a contradiction.

Thus $S$ is in convex position. If some three points of $S$ can be covered by only one homothet of $C$, then the whole $S$ must be covered by this homothet. We may therefore assume without loss of generality that any three points of $S$ are in more than one homothet of $C$. We now repeatedly apply Lemma 3 ,

Suppose conv $S$ has at least six vertices. Let $\boldsymbol{x}_{1}, \ldots, \boldsymbol{x}_{6}$ be any six vertices in this order on the boundary. The points $\boldsymbol{x}_{1}, \boldsymbol{x}_{3}, \boldsymbol{x}_{5}$ can be covered by more than one homothet of $C$. By Lemma 3 one of the segments $\left[\boldsymbol{x}_{1} \boldsymbol{x}_{3}\right],\left[\boldsymbol{x}_{3} \boldsymbol{x}_{5}\right],\left[\boldsymbol{x}_{5} \boldsymbol{x}_{1}\right]$ is contained in each homothet covering $\boldsymbol{x}_{1}, \boldsymbol{x}_{3}, \boldsymbol{x}_{5}$. If it is $\left[\boldsymbol{x}_{1} \boldsymbol{x}_{3}\right]$, then considering the homothet 
covering $\left\{\boldsymbol{x}_{1}, \boldsymbol{x}_{2}, \boldsymbol{x}_{3}, \boldsymbol{x}_{5}\right\}$ gives a contradiction, since $\left[\boldsymbol{x}_{1} \boldsymbol{x}_{3}\right]$ is then forced to be in the interior. A similar contradiction is obtained for the other two possibilities.

Thus conv $S$ has at most five vertices. If there are exactly five vertices $\boldsymbol{x}_{1}, \ldots, \boldsymbol{x}_{5}$ (in this order), then with the same reasoning as before, any homothet of $C$ containing e.g. $\left\{\boldsymbol{x}_{2}, \boldsymbol{x}_{4}, \boldsymbol{x}_{5}\right\}$ must contain $\left[\boldsymbol{x}_{4} \boldsymbol{x}_{5}\right]$. Thus any homothet of $C$ containing all vertices except $\boldsymbol{x}_{1}$ must contain $\left[\boldsymbol{x}_{2} \boldsymbol{x}_{3}\right] \cup\left[\boldsymbol{x}_{4} \boldsymbol{x}_{5}\right]$, etc. It follows that $C$ can be inscribed in a pentagon with sides parallel to $\boldsymbol{x}_{i} \boldsymbol{x}_{i+1}$. By the following lemma, this pentagon is homothetic to $\partial \operatorname{conv} S$, hence $S$ can be covered by a homothet of $C$.

Lemma 5. Consider two convex pentagons $\boldsymbol{x}_{1} \boldsymbol{x}_{2} \boldsymbol{x}_{3} \boldsymbol{x}_{4} \boldsymbol{x}_{5}$ and $\boldsymbol{x}_{1}^{\prime} \boldsymbol{x}_{2}^{\prime} \boldsymbol{x}_{3}^{\prime} \boldsymbol{x}_{4}^{\prime} \boldsymbol{x}_{5}^{\prime}$ with $\boldsymbol{x}_{i} \boldsymbol{x}_{i+1}$ parallel to $\boldsymbol{x}_{i}^{\prime} \boldsymbol{x}_{i+1}^{\prime}$ for each $i$. Assume that for each $i$, some homothet of $\left[\boldsymbol{x}_{i} \boldsymbol{x}_{i+1}\right] \cup\left[\boldsymbol{x}_{i+2} \boldsymbol{x}_{i+3}\right]$ is contained in $\left[\boldsymbol{x}_{i}^{\prime} \boldsymbol{x}_{i+1}^{\prime}\right] \cup\left[\boldsymbol{x}_{i+2}^{\prime} \boldsymbol{x}_{i+3}^{\prime}\right]$. Then the two pentagons are homothetic.

Proof of Lemma 可. Let $\alpha_{i}=\varangle \boldsymbol{x}_{i+1} \boldsymbol{x}_{i} \boldsymbol{x}_{i+2}, \beta_{i}=\varangle \boldsymbol{x}_{i-2} \boldsymbol{x}_{i} \boldsymbol{x}_{i+2}, \gamma_{i}=\varangle \boldsymbol{x}_{i-2} \boldsymbol{x}_{i} \boldsymbol{x}_{i-1}$, with similar definitions for $\alpha_{i}^{\prime}, \beta_{i}^{\prime}, \gamma_{i}^{\prime}$ (where indices are taken modulo 5). Then clearly $\gamma_{i}^{\prime} \leq \gamma_{i}$ and $\alpha_{i}^{\prime} \leq \alpha_{i}$ for all $i$. Since $\alpha_{i}+\beta_{i}+\gamma_{i}=\alpha_{i}^{\prime}+\beta_{i}^{\prime}+\gamma_{i}^{\prime}$ (the pentagons have parallel sides), it follows that $\beta_{i} \leq \beta_{i}^{\prime}$ for all $i$. Since $\sum_{i} \beta_{i}=180^{\circ}=\sum_{i} \beta_{i}^{\prime}$, we obtain $\beta_{i}=\beta_{i}^{\prime}$ for all $i$. Thus $\alpha_{i}+\gamma_{i}=\alpha_{i}^{\prime}+\gamma_{i}^{\prime}$ for all $i$, hence $\alpha_{i}=\alpha_{i}^{\prime}$ and $\gamma_{i}=\gamma_{i}^{\prime}$ for all $i$. Thus each triangle $\triangle \boldsymbol{x}_{i} \boldsymbol{x}_{i+1} \boldsymbol{x}_{i+2}$ is similar to $\triangle \boldsymbol{x}_{i}^{\prime} \boldsymbol{x}_{i+1}^{\prime} \boldsymbol{x}_{i+2}^{\prime}$. It follows that the pentagons are homothetic.

Proof of Theorem $B$, continued. We now consider the case where conv $S$ has exactly four vertices $\boldsymbol{x}_{1}, \boldsymbol{x}_{2}, \boldsymbol{x}_{3}, \boldsymbol{x}_{4}$ (labelled in this order). Since $\# S>4$, there is a point in the relative interior of some edge of conv $S$, say $\boldsymbol{y}_{1}$ between $\boldsymbol{x}_{1}$ and $\boldsymbol{x}_{2}$.

If there is a point in the relative interior of an edge adjacent to $\left[\boldsymbol{x}_{1} \boldsymbol{x}_{2}\right]$, say $\boldsymbol{y}_{2}$ between $\boldsymbol{x}_{2}$ and $\boldsymbol{x}_{3}$, then, by Lemma 3 one of the edges of $\boldsymbol{x}_{4} \boldsymbol{y}_{1} \boldsymbol{y}_{2}$ is contained in any homothet of $C$ containing $\boldsymbol{x}_{4}, \boldsymbol{y}_{1}, \boldsymbol{y}_{2}$. This leads to a contradiction as before. Thus there is no point in $S$ between $\boldsymbol{x}_{2}$ and $\boldsymbol{x}_{3}$, nor between $\boldsymbol{x}_{1}$ and $\boldsymbol{x}_{4}$.

There are now two cases:

1. There is no point between $\boldsymbol{x}_{3}$ and $\boldsymbol{x}_{4}$.

2. There is a point between $\boldsymbol{x}_{3}$ and $\boldsymbol{x}_{4}$.

In case 1, apply Lemma 3 to homothets of $C$ covering $\boldsymbol{x}_{1}, \boldsymbol{x}_{2}, \boldsymbol{x}_{4}$. If all such homothets cover $\left[\boldsymbol{x}_{1} \boldsymbol{x}_{2}\right]$, then we have found a homothet covering $S$. We may therefore assume without loss of generality that any homothet of $C$ covering $\boldsymbol{x}_{1}, \boldsymbol{x}_{2}, \boldsymbol{x}_{4}$ also covers $\left[\boldsymbol{x}_{1} \boldsymbol{x}_{4}\right]$, and similarly, any homothet of $C$ covering $\boldsymbol{x}_{1}, \boldsymbol{x}_{2}, \boldsymbol{x}_{3}$ also covers $\left[\boldsymbol{x}_{2} \boldsymbol{x}_{3}\right]$.

Thus any homothet of $C$ covering $\boldsymbol{x}_{1}, \boldsymbol{x}_{2}, \boldsymbol{x}_{4}, \boldsymbol{y}_{1}$ also contains $\left[\boldsymbol{x}_{1} \boldsymbol{x}_{4}\right] \cup\left[\boldsymbol{x}_{1} \boldsymbol{x}_{2}\right]$. Thus $C$ contains a wedge homothetic to $\left[\boldsymbol{x}_{1} \boldsymbol{x}_{4}\right] \cup\left[\boldsymbol{x}_{1} \boldsymbol{x}_{2}\right]$. Similarly, $C$ contains a wedge homothetic to $\left[\boldsymbol{x}_{1} \boldsymbol{x}_{2}\right] \cup\left[\boldsymbol{x}_{2} \boldsymbol{x}_{3}\right]$. It then easily follows that a homothet of $C$ covering $\boldsymbol{x}_{1}, \boldsymbol{x}_{2}, \boldsymbol{x}_{3}, \boldsymbol{x}_{4}$ must also cover $\left[\boldsymbol{x}_{1} \boldsymbol{x}_{4}\right] \cup\left[\boldsymbol{x}_{2} \boldsymbol{x}_{3}\right]$. If this homothet does not already cover $\left[\boldsymbol{x}_{1} \boldsymbol{x}_{2}\right]$, we may apply a further homothety to obtain a homothet covering $\left[\boldsymbol{x}_{1} \boldsymbol{x}_{2}\right] \cup\left[\boldsymbol{x}_{1} \boldsymbol{x}_{4}\right] \cup\left[\boldsymbol{x}_{2} \boldsymbol{x}_{3}\right]$.

In case 2 , let $\boldsymbol{y}_{2}$ be a point between $\boldsymbol{x}_{3}$ and $\boldsymbol{x}_{4}$. As in case 1 we obtain that one of the following two situations occurs:

(a) Any homothet of $C$ covering $\boldsymbol{x}_{1}, \boldsymbol{x}_{2}, \boldsymbol{x}_{4}$ also covers $\left[\boldsymbol{x}_{1} \boldsymbol{x}_{4}\right]$, and any homothet of $C$ covering $\boldsymbol{x}_{1}, \boldsymbol{x}_{2}, \boldsymbol{x}_{3}$ also covers $\left[\boldsymbol{x}_{2} \boldsymbol{x}_{3}\right]$.

(b) Any homothet of $C$ covering $\boldsymbol{x}_{1}, \boldsymbol{x}_{3}, \boldsymbol{x}_{4}$ also covers $\left[\boldsymbol{x}_{1} \boldsymbol{x}_{4}\right]$, and any homothet of $C$ covering $\boldsymbol{x}_{2}, \boldsymbol{x}_{3}, \boldsymbol{x}_{4}$ also covers $\left[\boldsymbol{x}_{2} \boldsymbol{x}_{3}\right]$. 
Assume without loss of generality that we have (a) (as in case 1). As before we have that $C$ contains homothets of $\left[\boldsymbol{x}_{1} \boldsymbol{x}_{4}\right] \cup\left[\boldsymbol{x}_{1} \boldsymbol{x}_{2}\right]$ and $\left[\boldsymbol{x}_{1} \boldsymbol{x}_{2}\right] \cup\left[\boldsymbol{x}_{2} \boldsymbol{x}_{3}\right]$. By Lemma 3 . any homothet of $C$ covering $\boldsymbol{x}_{2}, \boldsymbol{x}_{4}, \boldsymbol{y}_{2}$ must cover $\left[\boldsymbol{x}_{4} \boldsymbol{y}_{2}\right]$. Thus any homothet covering $\boldsymbol{x}_{1}, \boldsymbol{x}_{2}, \boldsymbol{x}_{4}, \boldsymbol{y}_{2}$ also covers the wedge $\left[\boldsymbol{x}_{1} \boldsymbol{x}_{4}\right] \cup\left[\boldsymbol{x}_{4} \boldsymbol{y}_{2}\right]$. Similarly, $C$ contains a homothet of $\left[\boldsymbol{x}_{2} \boldsymbol{x}_{3}\right] \cup\left[\boldsymbol{x}_{3} \boldsymbol{x}_{4}\right]$. Thus $C$ is a quadrilateral with sides parallel to the sides of conv $S$. As in case 1 there is a homothet of $C$ covering $\left[\boldsymbol{x}_{1} \boldsymbol{x}_{2}\right] \cup\left[\boldsymbol{x}_{1} \boldsymbol{x}_{4}\right] \cup\left[\boldsymbol{x}_{2} \boldsymbol{x}_{3}\right]$. It is then not difficult to see that there is a homothet of $C$ covering $\left[\boldsymbol{x}_{1} \boldsymbol{x}_{2}\right]$ and $\left[\boldsymbol{x}_{3} \boldsymbol{x}_{4}\right]$.

We now consider the case where conv $S$ has three vertices, say $\boldsymbol{x}_{1}, \boldsymbol{x}_{2}, \boldsymbol{x}_{3}$. If there are further points of $S$ on only one edge, say $\boldsymbol{y}$ between $\boldsymbol{x}_{1}$ and $\boldsymbol{x}_{3}$, then any homothet of $C$ covering $\left\{\boldsymbol{x}_{1}, \boldsymbol{x}_{2}, \boldsymbol{x}_{3}, \boldsymbol{y}\right\}$ contains $S$. If there are further points of $S$ on all three edges, then Lemma 3 applied to three such points, one from each edge, provides a contradiction. We may therefore assume that there are points $\boldsymbol{y}_{1}, \boldsymbol{y}_{2} \in S$ with $\boldsymbol{y}_{1}$ between $\boldsymbol{x}_{2}$ and $\boldsymbol{x}_{3}$ and $\boldsymbol{y}_{2}$ between $\boldsymbol{x}_{3}$ and $\boldsymbol{x}_{1}$, and no points of $S$ between $\boldsymbol{x}_{1}$ and $\boldsymbol{x}_{2}$.

Suppose that $\left[\boldsymbol{x}_{2} \boldsymbol{x}_{3}\right]$ is contained in any homothet of $C$ covering $\boldsymbol{x}_{1}, \boldsymbol{x}_{2}, \boldsymbol{x}_{3}$. Then any homothet covering $\boldsymbol{x}_{1}, \boldsymbol{x}_{2}, \boldsymbol{x}_{3}, \boldsymbol{y}_{2}$ covers all of $S$. A similar argument holds for $\left[\boldsymbol{x}_{1} \boldsymbol{x}_{3}\right]$. We may therefore assume that any homothet of $C$ covering $\boldsymbol{x}_{1}, \boldsymbol{x}_{2}, \boldsymbol{x}_{3}$ contains $\left[\boldsymbol{x}_{1} \boldsymbol{x}_{2}\right]$, by Lemma 3 Considering $\boldsymbol{x}_{1}, \boldsymbol{x}_{2}, \boldsymbol{x}_{3}, \boldsymbol{y}_{1}$, we see that $C$ has a wedge homothetic to $\left[\boldsymbol{x}_{1} \boldsymbol{x}_{2}\right] \cup\left[\boldsymbol{x}_{2} \boldsymbol{x}_{3}\right]$. Similarly, $C$ has a wedge homothetic to $\left[\boldsymbol{x}_{1} \boldsymbol{x}_{2}\right] \cup$ $\left[\boldsymbol{x}_{1} \boldsymbol{x}_{3}\right]$. Using Lemma 3 again, any homothet of $C$ covering $\boldsymbol{x}_{1}, \boldsymbol{x}_{2}, \boldsymbol{y}_{1}, \boldsymbol{y}_{2}$ must cover $\left[\boldsymbol{x}_{1} \boldsymbol{y}_{2}\right]$ and $\left[\boldsymbol{x}_{2} \boldsymbol{y}_{1}\right]$. Thus $C$ contains a homothet of $\left[\boldsymbol{x}_{1} \boldsymbol{y}_{2}\right] \cup\left[\boldsymbol{x}_{1} \boldsymbol{x}_{2}\right] \cup\left[\boldsymbol{x}_{2} \boldsymbol{y}_{1}\right]$. Any homothet of $C$ covering $\boldsymbol{x}_{3}, \boldsymbol{y}_{1}, \boldsymbol{y}_{2}$ must contain $\left[\boldsymbol{x}_{3} \boldsymbol{y}_{1}\right]$ or $\left[\boldsymbol{x}_{3} \boldsymbol{y}_{2}\right]$. Assume without loss of generality that it contains $\left[\boldsymbol{x}_{3} \boldsymbol{y}_{1}\right]$. Then any homothet containing $\boldsymbol{x}_{1}, \boldsymbol{x}_{3}, \boldsymbol{y}_{1}, \boldsymbol{y}_{2}$ must contain $\left[\boldsymbol{x}_{3} \boldsymbol{y}_{1}\right] \cup\left[\boldsymbol{x}_{3} \boldsymbol{x}_{1}\right]$. Thus $C$ is a triangle, a case that we excluded.

Finally, the case where conv $S$ has 2 vertices is trivial.

Proof of Theorem $A$. We again omit the simple proof that $\mathcal{H}_{\Delta}$ has Helly order 4, and assume for the remainder of the proof that $C$ is not the boundary of a triangle. We assume that $\bigcap \mathcal{S}=\emptyset$ and aim for a contradiction. Let $k+1$ be the smallest cardinality of a sub-collection of $\mathcal{S}$ with empty intersection. By assumption, $k \geq$ 4. Without loss of generality we may assume that $\mathcal{S}$ is this sub-collection, say $\mathcal{S}=\left\{C_{1}, \ldots, C_{k+1}\right\}$. For each $j=1, \ldots, k+1$, choose $\boldsymbol{x}_{j} \in \bigcap_{i \neq j} C_{i}$. Let $X=$ $\left\{\boldsymbol{x}_{1}, \ldots, \boldsymbol{x}_{k+1}\right\}$. Then $\boldsymbol{x}_{j} \notin C_{j}$ and any subset of $X$ of size $k$ contained in some $C_{i}$. By Theorem $\mathbb{B} X$ is contained in some homothet $C_{0}$ of $C$. Thus for all $i=1, \ldots, k+1, C_{0}$ and $C_{i}$ are two distinct homothets of $C$ covering $X \backslash\left\{\boldsymbol{x}_{i}\right\}$. Also, $X$ is in convex position.

If conv $X$ has at least six vertices, say $\boldsymbol{x}_{1}, \ldots, \boldsymbol{x}_{6}$ in this order, then, since $\boldsymbol{x}_{2}, \boldsymbol{x}_{4}, \boldsymbol{x}_{6}$ are contained in more than one homothet of $C$, one of $\left[\boldsymbol{x}_{2} \boldsymbol{x}_{4}\right],\left[\boldsymbol{x}_{4} \boldsymbol{x}_{6}\right]$, $\left[\boldsymbol{x}_{6} \boldsymbol{x}_{2}\right]$ must be contained in $C_{0}$, by Lemma 3. But $C_{0}$ also contains $\boldsymbol{x}_{1}, \boldsymbol{x}_{3}, \boldsymbol{x}_{5}$, contradicting its convexity.

If conv $X$ has exactly five vertices, say $\boldsymbol{x}_{1}, \ldots, \boldsymbol{x}_{5}$, then by Lemma 3 since $\boldsymbol{x}_{1}, \boldsymbol{x}_{2}, \boldsymbol{x}_{4}$ are contained in more than one homothet of $C,\left[\boldsymbol{x}_{1} \boldsymbol{x}_{2}\right] \subset C_{0}$. Similarly, $\left[\boldsymbol{x}_{i} \boldsymbol{x}_{i+1}\right] \subset C_{0}$ for all $i$. It follows that $C_{0}=\partial \operatorname{conv} X$. Again using Lemma 3 all homothets of $C$ containing $\boldsymbol{x}_{1}, \boldsymbol{x}_{2}, \boldsymbol{x}_{4}, \boldsymbol{x}_{5}$ must contain $\left[\boldsymbol{x}_{1} \boldsymbol{x}_{2}\right]$ and $\left[\boldsymbol{x}_{4} \boldsymbol{x}_{5}\right]$. But there is only one such homothet, viz. $C_{0}$ (since $C_{0}$ is the pentagon with vertices $\left.\boldsymbol{x}_{1}, \ldots, \boldsymbol{x}_{5}\right)$. This is a contradiction, since $C_{3}$ is another such homothet. 
If conv $X$ has exactly four vertices, say $\boldsymbol{x}_{1}, \ldots, \boldsymbol{x}_{4}$, then, since $\# X>4$, there must be an $\boldsymbol{x}_{5}$ on some edge, say on $\left[\boldsymbol{x}_{3} \boldsymbol{x}_{4}\right]$. Thus $\left[\boldsymbol{x}_{3} \boldsymbol{x}_{4}\right] \subset C_{0}$. Since $\boldsymbol{x}_{5} \notin C_{5}$, $\left[\boldsymbol{x}_{3} \boldsymbol{x}_{4}\right] \not \subset C_{5}$. Also, $\left[\boldsymbol{x}_{1} \boldsymbol{x}_{3}\right] \not \subset C_{0}$. Applying Lemma 3 to $\boldsymbol{x}_{1}, \boldsymbol{x}_{3}, \boldsymbol{x}_{4}$, we find that $\left[\boldsymbol{x}_{1} \boldsymbol{x}_{4}\right] \subset C_{0}$. Similarly, $\left[\boldsymbol{x}_{2} \boldsymbol{x}_{3}\right] \subset C_{0}$. Applying Lemma 3 to $\boldsymbol{x}_{1}, \boldsymbol{x}_{2}, \boldsymbol{x}_{5}$, we obtain that $\left[\boldsymbol{x}_{1} \boldsymbol{x}_{2}\right] \subset C_{0}$. It follows that $C_{0}=\partial \operatorname{conv} X$. Thus there is only one homothet containing $\boldsymbol{x}_{1}, \boldsymbol{x}_{2}, \boldsymbol{x}_{3}, \boldsymbol{x}_{4}$, a contradiction.

If conv $X$ has exactly three vertices, say $\boldsymbol{x}_{1}, \boldsymbol{x}_{2}, \boldsymbol{x}_{3}$, then, since $\# X \geq 5$, there must be at least two other points $\boldsymbol{x}_{4}, \boldsymbol{x}_{5}$. If they are on the same edge, say on $\left[\boldsymbol{x}_{1} \boldsymbol{x}_{2}\right]$, then, since $\boldsymbol{x}_{1}, \boldsymbol{x}_{2}, \boldsymbol{x}_{4} \in C_{5}$, we must also have $\boldsymbol{x}_{5} \in C_{5}$, a contradiction. Thus $\boldsymbol{x}_{4}$ and $\boldsymbol{x}_{5}$ are on different edges, say $\boldsymbol{x}_{4} \in\left[\boldsymbol{x}_{1} \boldsymbol{x}_{2}\right]$ and $\boldsymbol{x}_{5} \in\left[\boldsymbol{x}_{2} \boldsymbol{x}_{3}\right]$. Apply Lemma 3 to $\boldsymbol{x}_{1}, \boldsymbol{x}_{2}, \boldsymbol{x}_{3}$. If all homothets containing $\boldsymbol{x}_{1}, \boldsymbol{x}_{2}, \boldsymbol{x}_{3}$ also contain $\left[\boldsymbol{x}_{1} \boldsymbol{x}_{3}\right]$, then $C_{0}=\partial \operatorname{conv}\left\{\boldsymbol{x}_{1}, \boldsymbol{x}_{2}, \boldsymbol{x}_{3}\right\}$. Thus $C$ is a hollow triangle, a case we excluded. If all such homothets contain $\left[\boldsymbol{x}_{1} \boldsymbol{x}_{2}\right]$, then $\boldsymbol{x}_{4} \in C_{4}$, a contradiction. If all such homothets contain $\left[\boldsymbol{x}_{2} \boldsymbol{x}_{3}\right]$, then we have a similar contradiction.

Finally, if conv $X$ is collinear, then there are at least four points $\boldsymbol{x}_{1}, \boldsymbol{x}_{2}, \boldsymbol{x}_{3}, \boldsymbol{x}_{4}$ on a line, say with $\boldsymbol{x}_{2}, \boldsymbol{x}_{3}$ between $\boldsymbol{x}_{1}$ and $\boldsymbol{x}_{4}$. Since $\boldsymbol{x}_{1}, \boldsymbol{x}_{2}, \boldsymbol{x}_{4} \in C_{3}$, it follows that $\left[\boldsymbol{x}_{1} \boldsymbol{x}_{4}\right] \subset C_{3}$. But then $\boldsymbol{x}_{3} \in C_{3}$, a contradiction.

Our original assumption $\bigcap \mathcal{S}=\emptyset$ was therefore incorrect.

\section{ACKNOWLEDGEMENT}

The author thanks the referee for pointing out inaccuracies and incomplete proofs in a previous version of this paper.

\section{REFERENCES}

1. N. Amenta, Helly-type theorems and generalized linear programming, Discrete Comput. Geom. 12 (1994), 241-261. MR 96b:90035

2. L. M. Blumenthal, Theory and applications of distance geometry, second ed., Chelsea Publishing Co., New York, 1970. MR 42:3678

3. V. Boltyanski, H. Martini, and P. S. Soltan, Excursions into combinatorial geometry, SpringerVerlag, Berlin, 1997. MR 98b:52001

4. L. Danzer, B. Grünbaum, and V. Klee, Helly's theorem and its relatives, Convexity (V. L. Klee, ed.), Proc. of Symposia in Pure Math, vol. 7, A.M.S., 1963, pp. 100-181. MR 28:524

5. M. Deza and P. Frankl, A Helly type theorem for hypersurfaces, J. Combin. Theory Ser. A 45 (1987), 27-30. MR 88e:52012

6. J. Eckhoff, Helly, Radon, and Carathéodory type theorems, Handbook of Convex Geometry (P. M. Gruber and J. M. Wills, eds.), Elsevier Science Publishers B.V., 1993, pp. 389-448. MR 94k:52010

7. P. Frankl, Helly-type theorems for varieties, Europ. J. Combinatorics 10 (1989), 243-245. MR 90k:52014

8. A. Getmanenko, On orders of congruence of some sets in $\mathbf{R}^{n}$, unpublished manuscript.

9. A. Getmanenko, Helly-type theorems for plane convex curves, arXiv.org e-Print archive http://arxiv.org/abs/math.MG/0010311

10. B. Grünbaum, Borsuk's partition conjecture in Minkowski planes, Bull. Res. Council Israel. Sect. F 7F (1957/1958), 25-30. MR 21:2209

11. B. Grünbaum, Measures of symmetry for convex sets, Convexity (V. L. Klee, ed.), Proc. of Symposia in Pure Math, vol. 7, A.M.S., 1963, pp. 233-270. MR 27:6187

12. E. Helly, Über Mengen konvexer Körper mit gemeinschaftlichen Punkten, Jahresber. Deutsch. Math.-Verein. 32 (1923), 175-176.

13. H. Kramer and A. B. Németh, Equally spaced points for families of compact convex sets in Minkowski spaces, Mathematica (Cluj) 38 (1973), 71-78. MR 50:14504]

14. H. Maehara, Helly-type theorems for spheres, Discrete Comput. Geom. 4 (1989), 279-285. MR 90c:52016 
15. H. Martini, K. J. Swanepoel, G. Weiss, The geometry of Minkowski spaces - a survey. Part I, Expo. Math. 19 (2001) 97-142.

16. T. S. Motzkin, A proof of Hilbert's Nullstellensatz, Math. Zeitschr. 63 (1955), 341-344. MR 17:576f

17. K. J. Swanepoel, Helly-type theorems for hollow axis-aligned boxes, Proc. Amer. Math. Soc. 127 (1999), 2155-2162. MR 99k:52011

18. K. J. Swanepoel, Helly-type theorems for polygonal curves, Discrete Math. 254 (2002), 527537.

19. S. G. Wayment, On congruence indices for simple closed curves, Proc. Amer. Math. Soc. 28 (1971), 199-207. MR 43:1052

Department of Mathematics, Applied Mathematics and Astronomy, University of South Africa, P.O. Box 392, Pretoria 0003, South Africa

E-mail address: swanekj@unisa.ac.za 